\title{
A novel train rescheduling algorithm for correcting disrupted train operations in a dense urban environment
}

\author{
K. Kumazawa, K. Hara \& T. Koseki \\ Department of Electrical Engineering, Graduate School of Engineering, \\ The University of Tokyo, Japan
}

\begin{abstract}
Train rescheduling during disrupted service is a substantially significant task for urban railway operators. This task typically depends on the experiences and personal decisions of the professional operators. The operators use neither systematic methodologies for modifying train schedules nor quantitative criteria for measuring the quality of the rescheduled plans. Thus, operators have requested assistance in the form of a computer-aided train-rescheduling program. The authors have created a computer-aided train rescheduling system that seeks to minimize passenger inconvenience. The rescheduling algorithm calculates a value for the amount of inconvenience experienced by the passenger. In addition to a conventional passenger flow analysis, the authors' algorithm considers passenger overflow, which is defined as waiting time experienced by a passenger while queuing on a platform. The proposed rescheduling method also introduces the idea that the time required to create, broadcast, and implement a new plan of train operation is itself a delay which must be accounted for in order to render a realistic passenger flow analysis during disordered operation. The causality of train system events after the initial event that caused the disruption of service is discussed for a realistic analysis of passenger flow.
\end{abstract}

Keywords: train rescheduling, assistance system, evaluation.

\section{Introduction}

The rescheduling of train operations is often needed when a train operation is disordered by various malfunctions or accidents. The main tasks are presently 
undertaken by train dispatchers using their experience and intuition. It is very difficult, since there are many factors to be considered such as the location of trains and rolling stock, tracks and train layout, the location of drivers, passengers' demands, and so on. Corresponding to a high-speed and high-density train operation, dispatchers have requested an assistance system in order to reschedule train operation plans quickly and precisely.

The authors proposed a method to rate train operation plans quantitatively from the passengers' point of view. Based on this, the authors formulate an assistance system that chooses an appropriate method to modify a train schedule (Nagasaki et al. [1]).

In this paper, the authors propose a causality-based passenger flow estimation algorithm that reflects the altered behaviours of passengers during disrupted train operation. The passenger flow estimation algorithm calculates a value for the amount of inconvenience experienced by the passenger. In addition to a conventional passenger flow analysis, the authors' algorithm considers passenger overflow, which is defined as the waiting time experienced by a passenger while queuing on a platform.

Rescheduling train operation plans were assumed to be instantaneously made in a conventional model at the accident time. In fact, it takes time to make rescheduling train operation plans. In this paper, the authors propose a new passenger flow estimation method to obtain a realistic evaluation contents. This method also considers the time required to generate train rescheduling plans.

\section{Composition of train rescheduling system}

This computer-aided train rescheduling system makes a rescheduling plan based on the information of delay and various restrictions. The system consists of two main parts, one is creating a train plan and the other is evaluating the plan. In the part of creating a train plan, change of train diagram and determination of train arrival and departure are executed. In evaluating the plan, estimation of passengers' behaviour and calculation of evaluation indices are carried out. The train rescheduling plan is made by repeating process of adoption judgment of a new modification based on the evaluation indices. And the train rescheduling plan is presented to the train dispatchers. Figure 1 shows a composition of the computer-aided train rescheduling system.

\section{Creation of train operation plan}

Creating train operation plan in the system consists of application part of train rescheduling methods and decision part of arrival or departure time by simulating train operation.

\subsection{Constrains for train operation}

There are various constrains for train operation. In the train operation simulation, it is necessary to decide arrival or departure times that fulfil all severe conditions. 


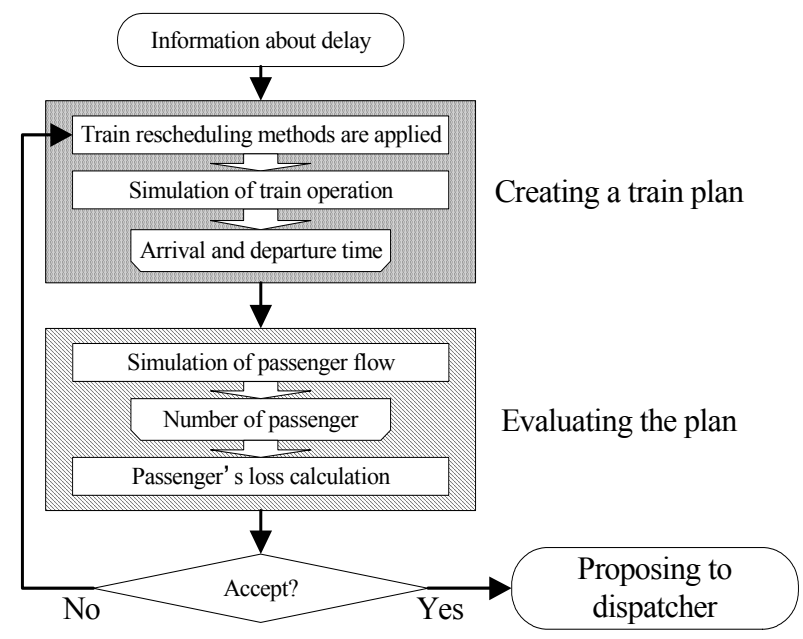

Figure 1: A composition of the computer-aided train rescheduling system.

The authors use graph structure to represent train operation. Nodes express departure and arrival of trains at each station. Links represent running and stopping of train, and constraints as follows.

\subsubsection{Regular arrival or departure time}

The arrival or departure time of each train in each station is provided beforehand. This schedule is a regular schedule. A train must not run earlier than a regular schedule.

\subsubsection{Regular running time}

To run between stations, a train requires longer time than regularly defined by the type of rolling stock, number of cars, stop or pass of stations, and so on.

\subsubsection{Regular stopping time}

A train stops at the station longer than the defined time. If the train does not stop at the station, the weight of the link equals to 0.

\subsubsection{Conflicting routes at a station}

A certain interval is required between trains whose routes conflict at a station or a yard. The weight of the link is time interval between departures and arrivals.

\subsubsection{Blockages between stations}

Trains more than defined number cannot run at the same time between stations.

\subsection{Simulation of train operation}

By finding the longest path to each node from the node expressing time origin, it is possible to obtain departure or arrival time of each train at each station as the length of the path. Also critical constraints in operating trains expressed by links framing the longest paths can be consequently obtained. 


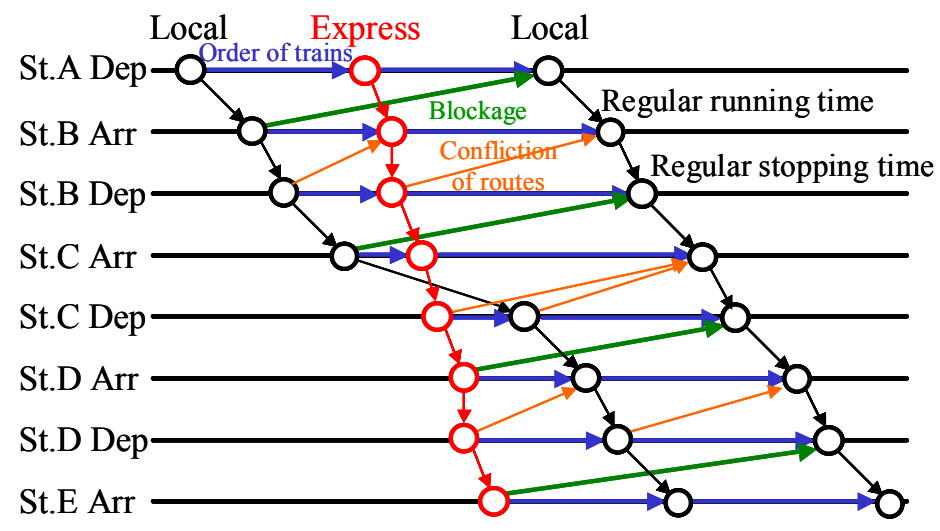

Figure 2: An example of a graph representing train operation.

The authors use PERT (Program Evaluation and Review Technique) for the longest path search (Abe and Araya [2]). It is necessary to change the information on nodes or links appropriately to express the application of train rescheduling techniques. Figure 2 shows an example of a graph representing train operation.

\section{Evaluation of train operation plan}

The total time of all trains' delay has been used for the evaluation of the train rescheduling plan in practical use. Because the delay is vanished when all delayed trains are suspension of service, it is evaluated as a good train rescheduling plan in this criterion. However, the passenger overflow on the platform actually, it is clear that this rescheduling plan is not good.

In order to avoid such stupid results, the authors has proposed a method to rate train operation plans quantitatively from passengers' point of view. This section describes how to estimate passenger flow and evaluation indices.

\subsection{Simulation of passenger flow}

The authors use graph and the shortest path search to decide lines and trains each passenger selects based on the models of passengers' behaviour. A node of a graph for calculating passenger flow expresses departure or arrival of a train at a station. A link describes potential passenger flow between departure and arrival. Figure 3 shows an example of a graph representing passenger flow.

\subsection{Graph for evaluating train operation plan from the passenger's point of view}

The authors defined a criterion for passengers' loss considering the following three evaluation indices, travelling time, burden of transfer, and congestion. These indices are calculated by using the result of simulation of passenger flow. 


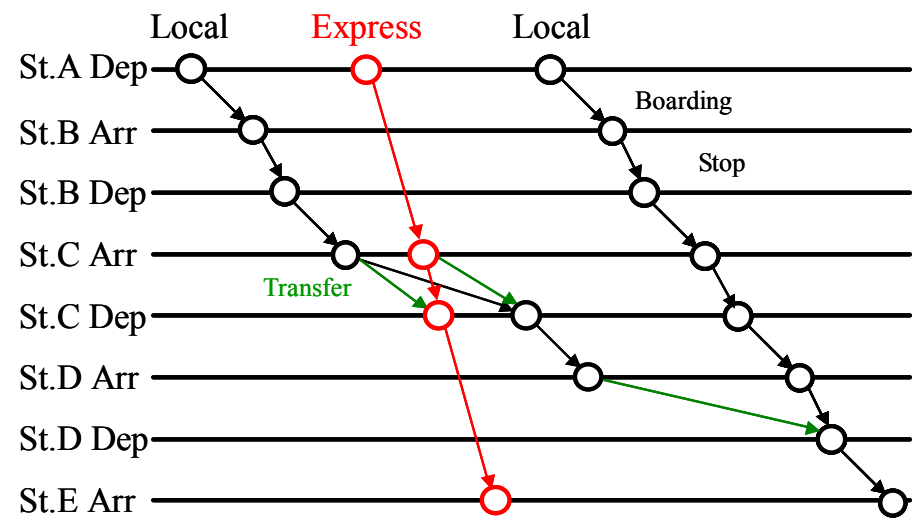

Figure 3: An example of a graph representing passenger flow.

\subsubsection{Travelling time}

The most significant factor for passengers to evaluate train operation is amount of time required from the starting station to the destination. Travelling time is defined as follows.

$$
L_{1}=\sum_{i=1}^{N} t_{i}
$$

where $\mathrm{N}$ is the number of passenger, $t_{i}$ is passenger $i$ 's travelling time.

\subsubsection{Transfers}

Transfer not only takes time but also imposes burden such as going upstairs. These burdens are calculated as passengers' loss in addition to the real time for transfer.

$$
L_{2}=\sum_{i=1}^{N} \sum_{j=1}^{M_{i}} r_{i j}
$$

where $M_{i}$ is the number of transfers passenger $i$ needs, $r_{i j}$ is time equivalent to the burden of passenger $i$ 's $j$-th transfer.

\subsubsection{Congestion}

Congestion of trains is also evaluated as loss because passengers in a congested train feel discomfort. Congestion loss is defined as follows.

$$
L_{3}=\sum_{k=1}^{n-1} \sum_{s=1}^{S_{k}} f_{c}\left(\frac{q_{k s}}{c_{k s}}\right) q_{k s} t_{k s}
$$

where $\mathrm{n}$ is the number of stations, $S_{k}$ is the number of trains which arrive at station k. $q_{k s}, t_{k s}$, and $c_{k s}$ are the number of passenger in the train, the time required, and the capacity of the train between $k$-th and $k+l$-th stations respectively. $f_{c}$ is a nonlinear function to convert passengers' discomfort in the congested train into equivalent time, as shown in Figure 4 (Mitani et al. [3]). 


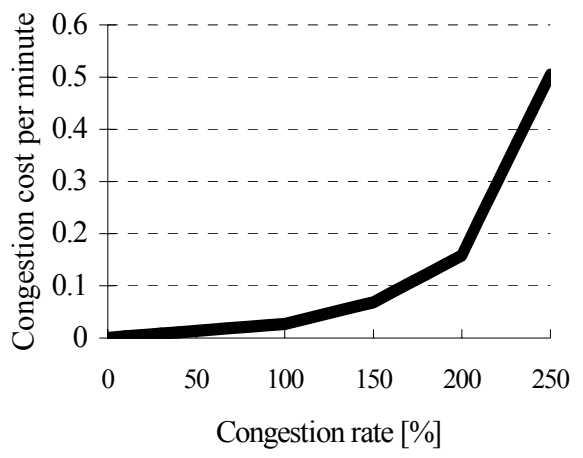

Figure 4: Relationship between congestion rate and congestion cost per minute.

The evaluation criterion of a train operation is the sum of above-mentioned three types of loss.

$$
L=L_{1}+L_{2}+L_{3}
$$

\section{Passenger overflow caused by congestion}

In the preceding study (Hara et al. [4]), passenger over flow by congestion was not considered. Therefore passengers could get on more than capacity of the train. This caused violence of causality. The passengers' behaviour was not correctly estimated and evaluation criteria could not be accurately calculated.

In this section, the authors model the process of passenger over flow by congestion.

\subsection{Model of passenger overflow}

Passenger overflow happens when there are more people on a platform waiting for a train than the rest capacity of next arriving train. For simplicity, the authors assume that all of the passengers on the platform are waiting in one line.

This simplification is important for calculating the point in time $t_{\mathrm{a}}$ when the number of waiting passengers exceeds the rest capacity of a next train. The point in time $t_{\mathrm{b}}$ when overflow occurs is determined by summation of the number of passengers arriving at a station after the preceding train has left the station. The number of passengers getting off and changing trains is added to this number of waiting passenger. The ratio of passengers able to successfully get on next train versus total passengers arriving at stations is assumed to be constant for each Origin-Destination pair. Passengers arriving at the station after the threshold for overflow point has been reach cannot get on the train they initially planed to board. These passengers must change their travel plans after the next train leaves the station. Figure 5 shows an example of a model of passenger overflow. 


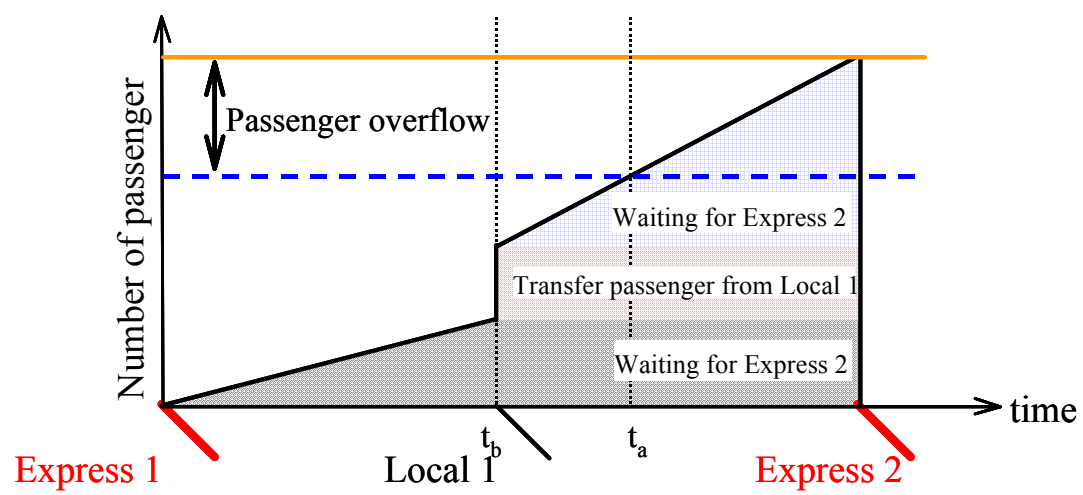

Figure 5: $\quad$ Model of passenger overflow.

\subsection{Effect of passenger overflow caused by congestion}

The authors have applied the proposed method to the diagram shown in Figure 6 in order to verify the effect. The capacity of each train is 800 people. Before the application of proposed method, there is the point where the number of the passengers exceeds the capacity of the train. This is not realistic. Applying the model of Figure 5, 70 passengers cannot take the train. These passengers take the next train. Figure 6 (b) is an application result of proposed method.

Figure 7 shows passengers' loss. Passenger overflow ultimately results in the numerical increase of passenger penalty function, as well increasing the individual passenger waiting time and inconvenience. This depends on the following reason, which passengers arriving at the station after the threshold for overflow has been reach cannot get on the train they initially planed to board. In detail, these passengers must adjust their travel plans after the next train leaves the station. So it takes more time to head for their destination. However, resolving some passenger overflow reduces the total passengers' loss. The congestion penalty in heavily loaded trains decreases drastically.

\section{Modelling of time necessary for planning and plan execution}

The authors proposed a method to rate train operation plans quantitatively from the passengers' point of view. Based on it, the authors formulated an assistance system that chooses an appropriate method to modify a train schedule.

However, rescheduling train operation plans were assumed to be instantaneously made in a conventional model at accident time (Hara et al. [4]).

In fact, it takes considerable time to make rescheduling train operation plans. In this section, the authors propose a new passenger flow estimation method to obtain a realistic evaluation index. This method considers also the time required to generate train rescheduling plans. 


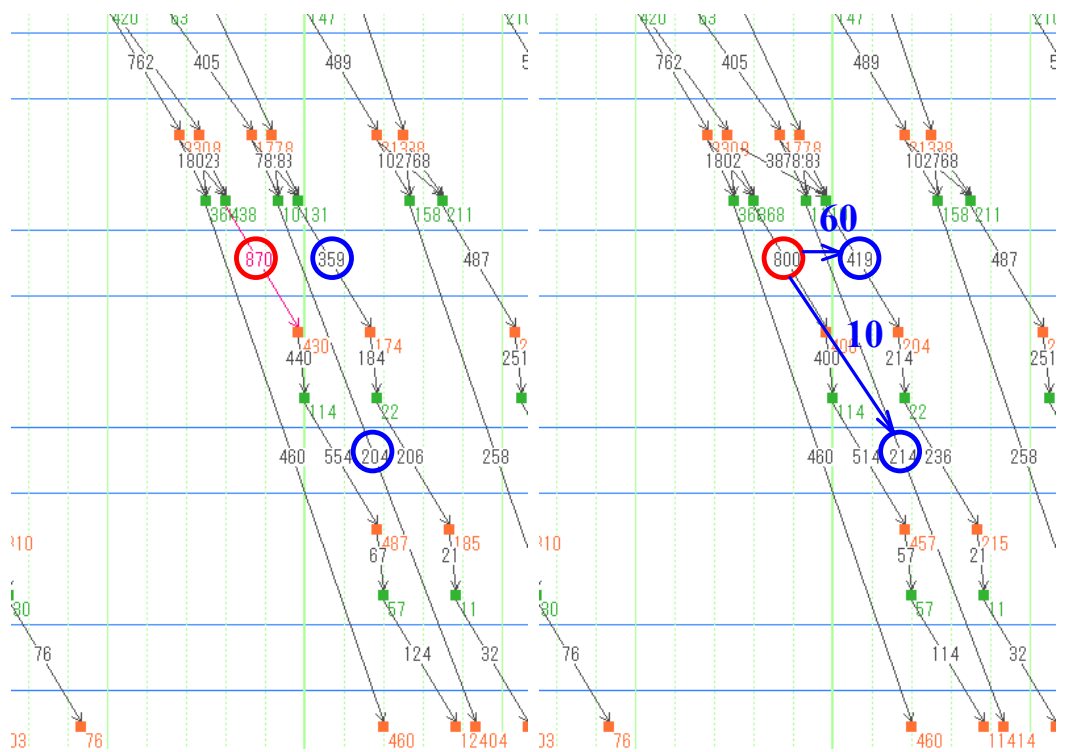

(a) Passenger overflow

(b) Apply this method

Figure 6: The proposed method applied to a diagram.

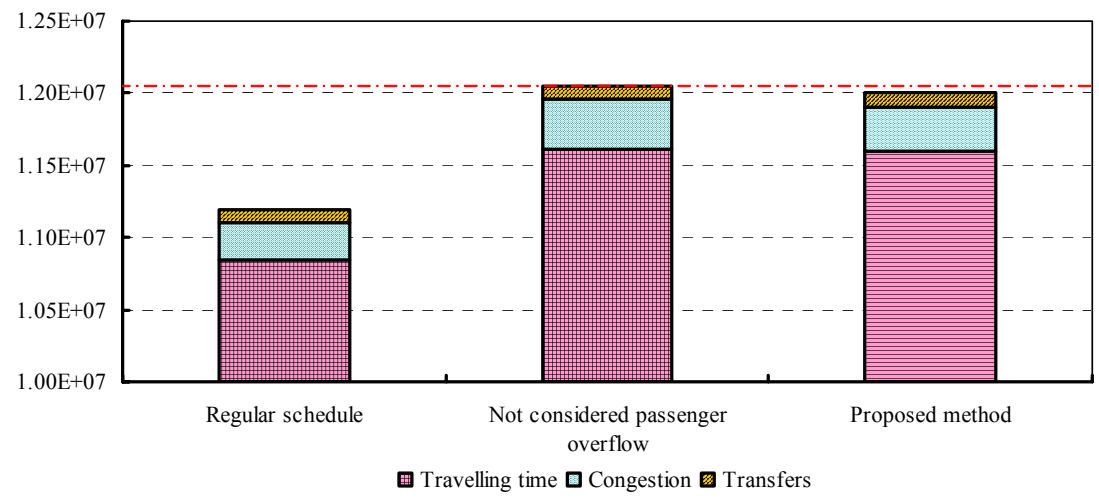

Figure 7: Comparison of passengers' loss.

\subsection{Modelling of time for planning and plan execution}

One needs a certain amount of time for planning and executing a new train schedule; it is impossible to realize a final rescheduling plan immediately after an operation disturbance. In order to take this planning time into consideration, the rescheduling algorithm is duelled into two instances beginning at two different points in time: the time when train service is initially disrupted and the 
time when the final rescheduling plan is started. A temporary train operation is inserted between the two times. This modification allows investigation of the effects of the time required for preparing the rescheduled plans. Figure 8 shows an example of a model of this method.

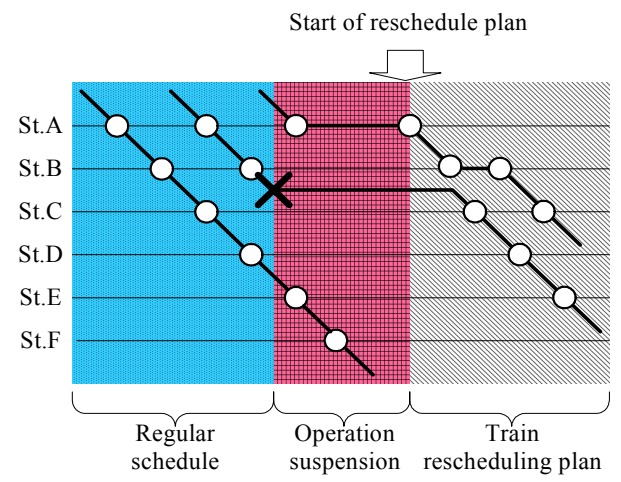

Figure 8: An example of a model of this method.

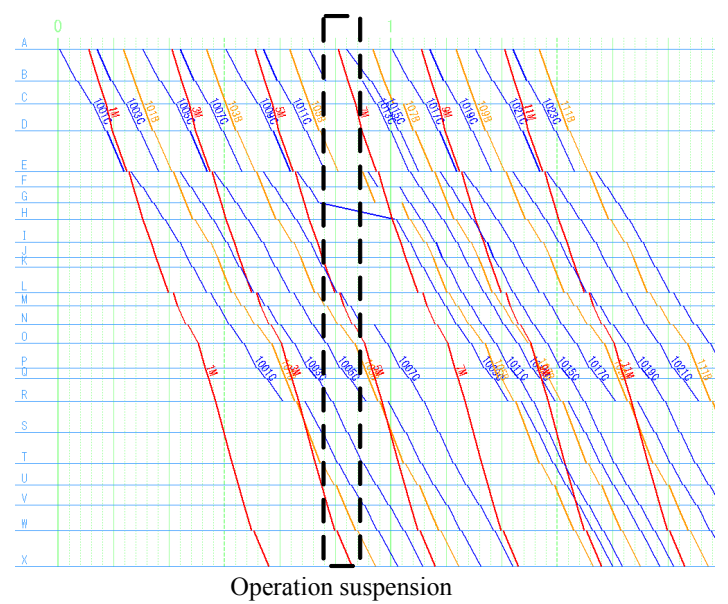

Figure 9: $\quad$ The proposed method applied to the diagram.

\subsection{Effect of special consideration given to the necessary for planning and its execution}

The authors have applied the proposed method to the diagram shown in Figure 9 in order to verify the effect. Operation suspension is done in a frame black in Figure 9. The time required to generate a train rescheduling plan can be expressible while operation suspension is done. By operation suspension being done, it turns out that passenger's loss increases. This depends on passenger's 
travelling time increasing. So this modification allows for the investigation of the effects of the time required for preparing the rescheduled plans.

\section{Conclusion}

Under a condition of rescheduling train operation plans were assumed to be instantaneously made in a conventional model at accident time (Hara et al. [4]), passenger overflow ultimately results in the numerical increase of passenger penalty function, as well increasing the individual passenger waiting time and inconvenience. However, in conjunction with the rest of the rescheduling system, allowing some passenger overflow reduces the total passenger penalty, including the congestion penalty, by decreasing congestion in heavily loaded trains.

In order to take this planning time into consideration, the rescheduling algorithm is duelled into two instances beginning at two different points in time. The two times are the time when train service is initially disrupted and the time when the final rescheduling plan is started. In this algorithm, it is not considered passenger overflow. The authors assert that their modification results in a more realistic evaluation of the passenger flow. However, it turns out that passengers' loss increases by passengers' travelling time increasing. So it is necessary to improve the temporary train.

\section{References}

[1] Nagasaki, Y., Eguchi, M. \& Koseki, T., Automatic Generation and Evaluation of Urban Railway Rescheduling Plan. Proc. of Int. Symp. on Speed-up and Service Technology for Railway and Maglev Systems STECH '03, Japan, 2003.

[2] Abe, K. \& Araya, S., Train Traffic Simulation using the Longest Path Method. Information Processing Society Journal, 1987 (in Japanese).

[3] Mitani, K., Ieda, H. \& Hatanaka, H., Evaluating Method of Congestion Cost by the Model of Passenger's Boarding Behavior. Infrastructure Planning Review (in Japanese), no. 5, pp. 139-146, 1987.

[4] Hara, K., Kumazawa, K. \& Koseki, T., Causality-based Passenger Flow Estimation in Irregular Train Operation for a Computer-aided Train Rescheduling System. Scheduling Symposium, pp.185-190, 2007 (in Japanese). 